\title{
Comet Shoemaker-Levy 9: Fragment and Progenitor Impact Energy
}

\author{
Toshiko Takata* and Thomas J. Ahrens ${ }^{+}$ \\ Lindhurst Laboratory of Experimental Geophysics, Division of Geological \& Planetary Sciences, California Institute of Technology, \\ Pasadena
}

Alan W. Harris

Jet Propulsion Laboratory, Pasadena, California

\begin{abstract}
Initial observational data from the impact of fragments of Comet Shoemaker-Levy 9 (SL9) are compared with smoothed particle hydrodynamic (SPH) calculations to determine their pre-impact diameters and the equivalent diameter of the SL9 progenitor. Diameters (solid ice) of 2.0 $\pm 0.1,2.0 \pm 0.05,2.1 \pm 0.04$ and $1.9 \pm 0.05 \mathrm{~km}$ for fragments $A$, E, G1, and $W$ are obtained from impact-induced plume heights from the Hubble Space Telescope (HST) data. Applying these values to scale apparent diameters for the balance of $18 \mathrm{SL}$ fragments in Weaver et al.'s [1995] catalog of 22 objects yields a SL9 progenitor diameter of $5.0 \pm 1.8 \mathrm{~km}$. This corresponds to total impact energy of $1.2(+1.8-0.8) \times 10^{30} \mathrm{erg}$. Such an energetic event occurs on Jupiter and Earth at least every 4,900 $+4,700,-2,700$, and $\sim 0.5 \times 10^{8}$ years, respectively.
\end{abstract}

\section{Introduction}

The most spectacular and energetic planetary event ever witnessed by humankind was the recent impact of fragments of Comet Shoemaker-Levy 9 (SL9) on Jupiter during the period of 16 to 21 July 1994 . Energy of SL9 fragments is the most important parameter required for understanding the impact phenomena and effects on the Jovian atmosphere. Although the velocity of the fragments $(60 \mathrm{~km} / \mathrm{s})$ was well determined from knowledge of the orbit [Yeomans and Chodos, 1993], actual fragment sizes and density were uncertain. Numerical models of Scotti and Melosh [1993] and Asphaug and Benz [1994], which are in part dependent on material properties of SL9, were used to reproduce the orbital position of the line of fragments after the break-up and indicated that the diameter of the parent body was $<2 \mathrm{~km}$ (diameter) and the total expected impact energy was $\sim 8$ $\times 10^{28} \mathrm{erg}$. In contrast, photometric measurements (with the HST) yielded an estimated (maximum) $7.4 \mathrm{~km}$ diameter parent body [Weaver et al., 1995] or total impact energy (solid ice) of 4 $\times 10^{30} \mathrm{erg}$. Thus there was an uncertainty of a factor of -50 for the total impact energy.

\section{Plume Height and and Impact Energy}

The height achieved by the SL9 fragment impact-induced shock-heated gas "plume" rising up in the inhomogeneous Jovian atmosphere is strongly dependent on impact energy and to a degree, studied, in part, by Crawford et al. [1995] and Boslough et al. [1995] on the effective diameter of the impacting

\footnotetext{
*Present address: Geological Institute, U. of Tokyo, Hongo, Bunkyoku, Tokyo, Japan

${ }^{+}$Correspondent
}

Copyright 1995 by the American Geophysical Union.

Paper number 95GL02237

0094-8534/95/95GL-02237\$03.00 fragment. Previous smoothed particle hydrodynamic (SPH) simulations [Ahrens et al., 1994; Takata et al., 1994] for the impact of solid ice fragments demonstrated that the impact energy deposited in the Jovian atmosphere resulted in plumes which rise to more than several tens of scale heights.

The plumes observed by Hammel et al. [1995] for fragments $A, E, G 1$, and W reached maximum heights of $2966 \pm 370$, $2916 \pm 170$ (lower bound), $3346 \pm 170$ and $2666 \pm 170 \mathrm{~km}$ (lower bound), respectively. The nearly constant height of these plumes (Fig. 1) is surprising as much larger variations in fragment sizes are inferred both from the pre-impact photometric data and in the variability of the area of opaque material deposited after impact of the SL9 fragments on the cloud tops. Here we used $0 \mathrm{~km}$ at the 1 bar datum. Interpolation of maximum plume heights between our SPH simulations [Takata et al., 1994] for a 0.4 and $2 \mathrm{~km}$ diameter bolide yields peak heights of -500 and $-3000 \mathrm{~km}$, respectively (Fig. 1) from:

$$
\mathrm{E} \text { (ergs) }=3.1 \times 10^{25} \mathrm{H}(\mathrm{km})
$$

where $E$ is impact energy and $H$ is plume height above the 480 $\mathrm{km}$ level. We assume in Eq. 1 that the plume has a ballistictype behavior for which the maximum height achieved is proportional to the kinetic energy per unit mass. This assumption needs further testing.

Since for a density of $1 \mathrm{~g} / \mathrm{cm}^{3}$ and impact velocity of 60 $\mathrm{km} / \mathrm{sec}, \mathrm{E}(\mathrm{erg})=9.4 \times 10^{27} \mathrm{D}^{3}(\mathrm{~km})^{3}$, it follows that

$$
\mathrm{D}(\mathrm{km})=0.15\left(\mathrm{H}^{\prime}(\mathrm{km})-459\right)^{1 / 3}
$$

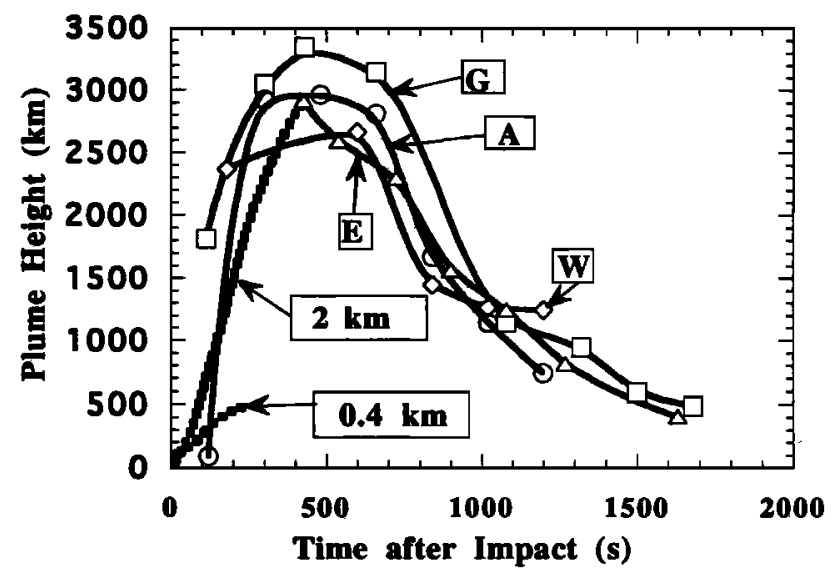

Figure 1. Plume height obtained from SPH calculations compared to HST [Hammel et al., 1995] observations. Three-dimensional SPH calculations give plume height for impact of $D=0.4$ and 2 $\mathrm{km}$ diameter at $60 \mathrm{~km} / \mathrm{sec}$ on Jupiter. Here, $0 \mathrm{~km}$ height represents the 1 bar pressure level. 
Table 1. Calculated diameter of 22 SL9 fragments. Diameters in first column are from the pre-impact photometric data [Weaver et al., 1995]. Fragment, A, G1, E and W diameters obtained from comparison of plume heights of our SPH simulations and the HST observations [Hammel et al., 1995]. Lower and upper limit values come from variance in plume heights observed from fragments and that derived from SPH calculation which is $3.1 \times 10^{25} \mathrm{erg} / \mathrm{km}$ above height of $479 \mathrm{~km}$.

\begin{tabular}{|c|c|c|c|c|}
\hline $\begin{array}{l}\text { Fragment } \\
\text { ID }\end{array}$ & $\begin{array}{l}\text { Upper Limit } \\
\text { diameter (km) } \\
\text { from Weaver } \\
\text { et al. }[1995]\end{array}$ & $\begin{array}{l}\text { Best estimate } \\
\text { diameter (km) } \\
\text { present work }\end{array}$ & $\begin{array}{l}\text { Lower Limit } \\
\text { diameter }(\mathbf{k m}) \\
\text { present work }\end{array}$ & $\begin{array}{l}\text { Upper Limit } \\
\text { diameter }(\mathrm{km}) \\
\text { present work }\end{array}$ \\
\hline$A^{*}$ & 1.4 & 2.01 & 1.91 & 2.11 \\
\hline $\mathbf{B}$ & 1.68 & 1.12 & 0.36 & 1.63 \\
\hline $\bar{C}$ & 2.10 & 1.40 & 0.45 & 2.04 \\
\hline D & 1.4 & 0.94 & 0.30 & 1.36 \\
\hline $\mathbf{E}$ & 2.8 & 2.00 & 1.95 & 2.04 \\
\hline$\overline{\mathbf{F}}$ & 2.1 & 1.40 & 0.45 & 2.04 \\
\hline G2 & 0.81 & 0.54 & 0.17 & 0.79 \\
\hline G1 & 4.06 & 2.11 & 2.07 & 2.15 \\
\hline $\mathbf{H}$ & 3.08 & 2.06 & 0.66 & 2.98 \\
\hline $\mathbf{K}$ & 3.78 & 2.53 & 0.81 & 3.66 \\
\hline $\mathbf{L}$ & 3.50 & 2.34 & 0.75 & 3.39 \\
\hline $\mathbf{N}$ & 1.4 & 0.94 & 0.30 & 1.36 \\
\hline P2 & 1.96 & 1.31 & 0.42 & 1.90 \\
\hline P1 & 0.854 & 0.57 & 0.18 & 0.83 \\
\hline Q2 & 3.08 & 2.06 & 0.66 & 2.98 \\
\hline Q1 & 4.06 & 2.71 & 0.87 & 3.93 \\
\hline $\mathbf{R}$ & 2.52 & 1.68 & 0.54 & 2.44 \\
\hline $\mathbf{S}$ & 2.94 & 1.96 & 0.63 & 2.85 \\
\hline $\mathbf{T}$ & 0.644 & 0.43 & 0.14 & 0.62 \\
\hline $\mathbf{U}$ & 0.91 & 0.61 & 0.20 & 0.88 \\
\hline $\mathbf{V}$ & 1.36 & 0.91 & 0.29 & 1.32 \\
\hline $\mathbf{w}$ & 2.38 & 1.93 & 1.88 & 1.98 \\
\hline Total -22 & 7.43 & 4.98 & 3.20 & 6.79 \\
\hline $\begin{array}{l}\text { fragments, } \\
\text { progenitor } \\
\text { diameter }(\mathrm{km})\end{array}$ & & & & \\
\hline
\end{tabular}

*Bold face: Indicates plume heights reported by Hammel et al . [1995].

where $\mathrm{H}^{\prime}$ is altitude measured from the 1 bar level. Applying this to observed plume heights gives DSPH $=2.0 \pm 0.1,2.0 \pm 0.05$, $2.1 \pm 0.04$, and $1.9 \pm 0.5 \mathrm{~km}$ for $\mathrm{A}, \mathrm{E}, \mathrm{Gl}$, and W. Upper limits on the diameter of each fragment, $\mathrm{DW}$, are available from Weaver et al. [1995] optical data. We assume that the squares of these upper limits are proportional to the observed brightness of each fragment. This assumption would be closely valid if the brightness is proportional to disc area of each fragment. For each of fragments $A, E, G 1$, and $W$, then, we can calculate an area scaling parạmeter.

$$
\mathrm{S}_{\mathrm{a}}=\left(\mathrm{D}_{\mathrm{SPH}} / \mathrm{D}_{\mathrm{w}}\right)^{2}
$$

which represents our best estimate of the ratio of the true area (inferred from the SPH simulations and the observed plume heights) to the effective photometric area; we obtain $S_{a}=2.3$, $0.53,0.28$, and 0.69 , respectively, with an average value of 0.94 \pm 0.90 . Thus, the diameter of each the 18 fragments whose plumes were not observed by Hammel et al. [1995], but whose brightness was obtained by Weaver et al. [1995], can be estimated to lie between 0.2 and 1.36 times $D_{W}$, with $\sqrt{0.94}=0.97 \mathrm{D}_{\mathrm{W}}$ as the best estimate (column 3 of Table 1). Additional observational (HST) uncertainties for A, E, G1, and $W$ are reflected in Table 1 (columns 4 and 5). Since the balance of the fragment diameters were scaled with the average of the 4 scaling parameters calculated from Eq. 3, the uncertainties are dominated by the uncertainties in $S_{a}$.

The plume gas ejected above the stratosphere follows a $\sim 10^{1}$ minute parabolic trajectory. Re-impact at a maximum radius of $\sim 4 h$ from the ejection point, where $h$ is the maximum plume height, yields a radial range of the ejecta of $-12,000 \mathrm{~km}$ for the G1 impact. This agrees with the radius $(10,000-14,000 \mathrm{~km})$ for the crescent-shaped dark region observed after impact of fragment G1 [Hammel et al., 1995]. However, the reasons the other impacts, for which plumes achieved similar heights, did not produce as large a radius dark regions, is not understood. Recently, Boslough et al. [1995] have suggested a model of this process.

We compare our SPH calculations to the HST data for the A, E, G1, and W plume heights (Fig. 1) to determine the diameter of $A, E, G 1$, and W fragments, and then to scale Weaver's et al. [1995] catalog (Table 1). This yields $5.0 \pm 1.8 \mathrm{~km}$ for an (ice) comet progenitor diameter. The corresponding equivalent energy of a solid ice projectile at $60 \mathrm{~km} / \mathrm{sec}$ is $1.2(1.8,-0.8) \mathrm{x}$ $10^{30}$ ergs.

\section{Detection of Deep Atmospheric Constituents}

Prior to the SL9 impact on Jupiter, it was widely believed that these impacts would exhume $\mathrm{H}_{2} \mathrm{O}$ from below its presumed 3 to 5 bar pressure level cloud deck and possibly $\mathrm{H}_{2} \mathrm{O}$ from deeper in 
the planet. Both finite difference [Boslough et al., 1994; Mac Low and Zahnle, 1994; Zahnle and MacLow, 1994] and our [Ahrens et al., 1994; Takata et al., 1994] SPH calculations agree that $-8 \times 10^{28} \mathrm{erg}$ (2 $\mathrm{km}$ diameter) impactors penetrate Jupiter to pressures of $-30 \mathrm{bar}$ or depths of $170 \mathrm{~km}$ below the 1 bar level. The HST team detected abundant absorption features corresponding to $\mathrm{NH}_{2}, \mathrm{~S}_{2}, \mathrm{CS}_{2}$, and $\mathrm{H}_{2} \mathrm{~S}$ and emission from $\mathrm{Mg}, \mathrm{Mg}^{+}, \mathrm{CS}$ and Fe [Noll et al., 1995]. Although, no waterderived species are presently reported as being detected at any ground-based observational site Bjorker et al. [1994] using the Kuiper Airborne Observatory, detected emission from $\mathrm{H}_{2} \mathrm{O}$ after the $\mathbf{G 1}$ and $\mathrm{K}$ impacts for about a half-hour and concluded that SL9 was indeed cometary. The spatial distribution of atmospheric gas within the plume from a $2 \mathrm{~km}$ fragment, some 2 minutes after impact (Figure 2), indicates that at this point, the stratospheric plume has risen to an altitude of $900 \mathrm{~km}$. The atmosphere originally below the expected water cloud deck has risen to an altitude of only $270 \mathrm{~km}$. Previously, published [Takata et al., 1994] velocity field results indicate that this lower altitude material is lofted at a much lower velocity than the material at the top of the plume. Also, gaseous material along the trajectory of the $\mathbf{2} \mathrm{km}$ comet fragment, that contains most of the vaporized cometary material, has risen to only an altitude of $-300 \mathrm{~km}$. Similarly, the lateral diameter of the plume is $\sim 10^{3}$ $\mathrm{km}$, whereas, the material from the expected water cloud has expanded only $-300 \mathrm{~km}$ laterally. Thus, we expect on the basis of this work that only a small fraction of the material at the level of the water clouds or material from the comet could be directly observed. Never-the-less, it appears that Bjorker et al. did observe exhumed water either of cometary or deep Jovian origin.

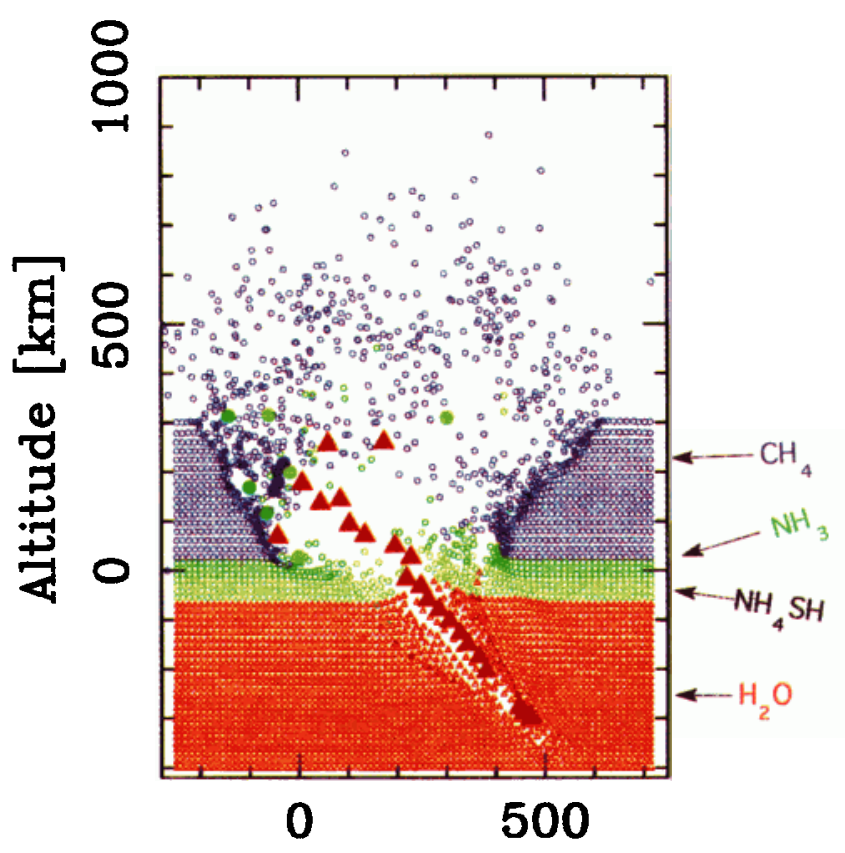

\section{Distance [km]}

Figure 2. SPH calculated cross-section of plume expansion from impact of $2 \mathrm{~km}$ diameter fragment (at $60 \mathrm{~km} / \mathrm{sec}$ ) after 123 seconds. Large symbols indicate material along bolide trajectory which contains cometary debris and the colors and symbols indicate at which levels these particles originated.
Infrared observations [Orton et al., 1995] indicate that the temperature of the impact sites, from a structure modeled as $0.25-\mu \mathrm{m}$ particles, for the $G, L$ and $Q$ features at the 1 to 200 mbar pressure levels did not change appreciably after the impact, and hence, it is claimed that SL9 fragments may not have penetrated to these pressure levels. However, although the atmospheric plume radii observed exceed $2 \times 10^{3} \mathrm{~km}$ for many of the impacts, the lateral "plume stalk" within the troposphere according to our calculations expanded to less than $300 \mathrm{~km}$ even for the largest ( $2 \mathrm{~km}$ diameter) impactors. We infer that available telescopic resolution was simply unable to detect radiation from deeper constituents coming from a restricted $\left(10^{2}\right.$ $\mathrm{km}$ ) area, or the radiation from lofted water was blocked by the opacity of the stratospheric plume. The depths, $d$, at which various energy impactors deposit their peak energies, from the calculations of Takata et al. [1994], MacLow and Zahnle [1994], and Zahnle and MacLow [1994] are given by

$$
d(\mathrm{~km})=-2100+80\left(\log _{10} \mathrm{E}(\mathrm{erg})\right)
$$

For $E=7.5 \times 10^{28}$, Eq. 4 yields a depth of $150 \mathrm{~km}$. We conclude that penetration at least to the -100 bar level did occur upon impact of $2 \mathrm{~km}$ fragments of SL9. Field and Ferrara [1995] point out that even if SL9 fragments were initially porous $\left(0.2 \mathrm{~g} / \mathrm{cm}^{3}\right)$, they would be fully compacted by the time they reached the 1 to 10 bar pressure level. We conclude that deep atmospheric gas and vaporized cometary debris exists within the lower $1 / 3$ of the impact induced plumes and it is possible that Jovian water remained deep inside the plumes, and thus was not visible to observers after plume cooling and subsequent collapse [Boslough et al., 1994].

\section{Frequency of SL9 Events on Jupiter and Earth}

Recently, Hockey [1994] examined the historical record of possible previous impact events on Jupiter since W. Hershel presented the first drawings of equatorial features in 1781 . Hockey concluded that no feature as large as the G1 impact site has previously been reported since the Hershel drawings. Chapman and Morrison [1994] have also reexamined the impact flux on Earth. These studies then motivate the question, how frequently do SL9 energy events occur on Jupiter versus the Earth? We first note that the impactor populations for the two planets are different -..-- being largely short-period (mostly extinct) comets for Jupiter and some $75 \%$ asteroids and $25 \%$ comets for the Earth. Recently, Shoemaker et al. [1995] concluded that SL9 was probably an extinct Jupiter family comet and that some 3000 comets with progenitor diameters, $\mathrm{D}_{\mathrm{p}}$, greater than $2.5 \mathrm{~km}$, again largely extinct per year (largely short-period) have perihelia within Jupiter's orbit. Using the magnitude-frequency relations of Shoemaker and Wolfe [1990] this flux

$$
F_{j p}=3000\left(2.5 / D_{p}\right)^{2}
$$

would be slightly lower for comets larger than the $5.0 \pm 1.8 \mathrm{~km}$ SL9 progenitor obtained here to a flux, $F_{j p}$, of $750(+950,-370)$ comets per year. Jupiter's effective impact cross-section is given by

$$
A_{j}=A_{j d}\left(1+\left(V_{e s c} / V_{\infty o}\right)^{2}\right)
$$

where $A_{j d}$ is the geometric area of the Jovian disc, $V_{\text {esc }}$ is the Jovian escape velocity, $57.2 \mathrm{~km} / \mathrm{sec}$, and $V_{\infty}$ is the assumed 
approach velocity far from Jupiter. Using a value of $\mathrm{V}_{\infty}$ of 10 $\mathbf{k m} / \mathbf{s e c}$, appropriate for short-period comets at Jupiter, yields a value of $A_{j}$ of $5.2 \times 10^{21} \mathrm{~cm}^{2}$, some 34 times $A_{j d}$. The annual rate of short-period comet impacts on Jupiter per year is thus

$$
F_{j}=F_{j p} A_{j} / A_{j s}
$$

where $A_{j}$ is the area of a sphere with a radius equal to the Jovian orbital radius. If SL9 was originally an extinct shortperiod comet, the time between SL9-sized impacts (assuming a single bolide) on Jupiter is $\mathrm{F}_{\mathrm{j}}{ }^{-1}=4,900(+4700,-2700)$ years.

The impact of G1-sized impactors is, of course, predicted to be more frequent. These occur every $880 \pm 35$ years. As no G1sized impact has been observed on Jupiter in the last 213 years, this result is consistent with our calculations.

In contrast, on the Earth, an object with the energy of the progenitor of SL9, or $1.2(+1.8,-0.8) \times 10^{30} \mathrm{ergs}(5.0 \mathrm{~km}$ diameter ice sphere, impacting at $60 \mathrm{~km} / \mathrm{sec}$ ) is rare and is likely to correspond only to a long-period comet. Weissman [1990] estimates that only some $8 \%$ of the objects impacting the Earth, in this energy range, are long-period comets. The most probable $1.2 \times 10^{30}$ erg impactors on the Earth, are the near-earth asteroids and their most probable impact velocity is $-20 \mathrm{~km} / \mathrm{sec}$. Recently, Chapman and Morrison [1994] estimated that a $1.2 \mathrm{x}$ $10^{30} \mathrm{erg}$ (equivalent to $3 \times 10^{7}$ Mtons of TNT) impactor strikes the Earth at intervals of $0.5 \times 10^{8}$ years. Moreover, they point out that such an event, although unlikely to cause complete planetary sterilization, is likely to lead to massive extinctions and a major global catastrophe as it is comparable to the energy of the Cretaceous-Tertiary extinction impactor.

In conclusion, we note that a SL9-energy impactor on Jupiter is $-10^{4}$ times more likely than on Earth.

Acknowledgments. We thank Glenn Orton, James Friedson, Andrew Ingersoll, Reta Beebe, Heidi Hammel, Paul R. Weissman and the HST team for directing us to data and helpful discussion, and Eugene $M$. Shoemaker, Paul R. Weissman, Carolyn S. Shoemaker, George Field, and A. Ferrara for their preprints. We thank reviewer, M. Boslough, an anonymous reviewer, and $W$. Anderson for comments on the manuscript. Research supported by NASA. Contribution No. 5462. Division of Geological and Planetary Sciences, California Institute of Technology.

\section{References}

Ahrens, T. J. et al., Impact of Comet Shoemaker-Levy 9 impact on Jupiter, Geophys. Res. Lett., 21, 1087-1090, 1994.

Asphaug, E., and W. Benz, Density of Comet Shoemaker-Levy 9 deduced by modeling breakup of the parent rubble-pile, Nature, 370, 120-124, 1994.

Bjoraker, G. L. et al., Detection of water in the fireball of fragments $G$ and $k$ of Comet Shoemaker-Levy 9, Bull. Amer. Astron., 26, 1585, 1994.

Boslough, M. B. et al., Mass and penetration depth of Shoemaker-Levy
9 fragments from time resolved photometry, Geophys. Res. Lett., 21 , 1555 - 1558, 1994.

Boslough, M. B. et a!., Numerical modeling of Shoemaker-Levy 9 impacts as a framework for interpreting observations, Geophys. Res. Lett., 22, 1821-1824, 1995.

Chapman, C. R., and D. Morrison, Impacts on the Earth by asteroids and comets: assessing the hazard, Nature, 367, 33-40, 1994.

Crawford, D. A. et al., The impact of periodic comet Shoemaker-Levy 9 on Jupiter, Int. J. Impact Eng., in press, 1995.

Field, G. B., and A. Ferrara, The behavior of fragments of Comet Shoemaker-Levy-9 in the atmosphere of Jupiter, Astrophys. J., 438, 957-967, 1995.

Hammel, H. B. et al., Hubble space telescope imaging of Jupiter: Atmospheric phenomena created by the impact of Comet ShoemakerLevy-9, Science, 267, 1288-1296, 1995.

Hockey, T., The Shoemaker-Levy 9 spots on Jupiter: Their place in history, Earth, Moom and Planets, 66, 1-10, 1994.

MacLow, M. M., and K. Zahnle, Explosion of Comet Shoemaker-Levy9 on entry into the Jovian atmosphere, Astrophys. J., 434, 33-44, 1994.

Noll, K. S. et al., HST spectroscopic observations of Jupiter after the collision of Comet Shoemaker-Levy-9, Science, 267, 1302-1305, 1995.

Orton, G. et al., The NASA infrared telescope facility investigation of Comet Shoemaker-Levy 9 and its collision with Jupiter: Preliminary results, Science, 267, 1277-1282, 1995.

Scotti, J. V., and H. J. Melosh, Tidal breakup and dispersion of P/Shoemaker-Levy 9: Estimate of progenitor size, Nature, 365, 733 735, 1993.

Shoemaker, E. M., P. R. Weissman, and C. S. Shoemaker, The flux of periodic comets near the Earth, in Hazards due to Comets and Asteroids, edited by T. Gehrels, pp. 313-336, U. Ariz., 1995.

Shoemaker, E. M., R. F. Wolfe, and C. S. Shoemaker, Asteroid and comet flux in the neighborhood of Earth, in Global Catastrophes in Earth History, vol. 247, edited by V. L. Sharpton and P. D. Ward, pp. 155-170, Geol. Soc. Am. Sp. Paper, 1990.

Takata, T. et al., Comet Shoemaker-Levy-9: Impact on Jupiter and plume evolution, Icarus, 109, 3-19, 1994.

Weaver, H. A. et al., The Hubble Space Telescope (HST) Observing Campaign on Comet Shoemaker-Levy 9, Science, 267, 1282-1288, 1995.

Weissman, P. R., The cometary impactor flux at the Earth, in Global Catastrophes in Earth History, vol. 247, edited by V. L. Sharpton and P. D. Ward, pp. 171-180, Geol. Soc. Amer. Sp. Paper, 1990.

Yeomans, D. K., and P. Chodos, Periodic Comet Shoemaker-Levy 9 (1993e), IAU Circ., 5909, 1, 1993.

Zahnle, K., and M. M. MacLow, The Collision of Jupiter and Comet Shoemaker-Levy 9, Icarus, 108, 1-17, 1994.

T. Takata and T. J. Ahrens, Lindhurst Laboratory of Experimental Geophysics, Division of Geological \& Planetary Sciences, California Institute of Technology, Pasadena CA 91125 (e-mail: tja@caltech.edu)

A. W. Harris, Jet Propulsion Laboratory, Pasadena, CA 91109 (email: awharris@lithos.jpl.nasa.gov)

(Received: February 16, 1995 revised: June 26, 1995 accepted: July 10, 1995) 